Paper can be cited as:

Fernandez, E. J. (in press). Training as enrichment: A critical review. Animal Welfare.

Check Animal Welfare or Google Scholar for publication updates.

\title{
Training as Enrichment: A Critical Review
}

\author{
Eduardo J. Fernandez
}

School of Animal and Veterinary Sciences, The University of Adelaide

\begin{abstract}
Husbandry training and environmental enrichment are both important advancements associated with current behavioral welfare practices. Additionally, the use of training procedures has been proposed as a form of enrichment, with the implication that training can produce beneficial behavioral welfare results. This paper examines the concept of training as enrichment through three distinct ways training procedures could enrich: (1) training facilitates enrichment usage, (2) training modifies interactions, conspecific or otherwise, and (3) training expands behavioral repertoires. Within each category, the paper focuses on past research that provides empirical support for training functioning as enrichment, as well as related areas of research that provide additional evidence. Previous studies support the claim that training is enriching, with additional research necessary to better understand how prevalent and under what conditions training procedures function as enrichment. Future training research should examine these potential enrichment effects, including methodology that allows for comparisons to traditional enrichment, the use of welfare diversity/variability indices, and the effects of learning on trainers and trainees alike.
\end{abstract}

Keywords: behavior analysis; enrichment; husbandry; learning; positive reinforcement; training; welfare

\section{Introduction}

The modern existence of animals under human care is connected to two major behavioral welfare advances: The use of animal training procedures to promote the husbandry of animals (Desmond \& Laule, 1994; Forthman \& Ogden, 1992; Laule et al., 2003; Melfi et al., 2020), and the implementation of environmental enrichment (Maple \& Perdue, 2013; Markowitz, 1982; Shepherdson et al., 1998; Young, 2003). Animal training can be defined by respondent and operant conditioning procedures used to elicit, 
evoke, or emit behavior (Domjan, 2014; Pierce \& Cheney, 2013; Pryor, 1999; Pryor \& Ramirez, 2014; Ramirez, 2020). For instance, in the case of an operant conditioning procedure, food or some other consequence is delivered as a reward for engaging in a selected response. It is also worth noting that most of the focus on modern animal training presented within this review emphasizes positive reinforcement and similar force-free applications to effectively change behavior. Environmental enrichment can be defined as stimuli and/or events that are added to or modify an animal's environment and result in some measurable improvement in behavioral and/or physiological wellbeing/welfare (Fernandez et al., 2021a; Fernandez \& Timberlake, 2008; Hoy et al., 2010; Mellen \& MacPhee, 2001; Newberry, 1995; Shepherdson, 1998). Some examples of enrichment include the use of foraging devices and feeding schedules, both automated and non-automated (Andrews \& Ha, 2014; Bashaw et al., 2016; Fernandez, 2010; Fernandez, 2021; Carlstead et al., 1991; Shepherdson et al., 1993), changes in enclosure presentations, including choice between enclosures (Carlstead et al., 1993; Coe, 2004; Sherwin et al., 1999), and the presentation of auditory, olfactory, and/or visual stimuli (Carlstead \& Seidensticker, 1991; Fernandez \& Timberlake, 2019a; Graham et al., 2005; Platt \& Novak, 1997; Wells \& Irwin, 2008).

While both training and enrichment advances have remained relatively autonomous, the concept of training as a form of enrichment itself has been proposed (Brando, 2012; Laule, 2003; Laule \& Desmond, 1998; Laule \& Whittaker, 2007; Melfi, 2013; Melfi, 2014; Melfi \& Ward, 2020; Westlund, 2014). The implication is that enrichment is a means to improve the welfare of captive animals, and training is proposed to improve welfare, and is therefore enriching. However, what remains less clear are the ways animal training procedures could be empirically measured to have an enriching effect. Some authors, such as Laule and Desmond (1998) and Westlund (2014) have proposed training enriches by providing animals with greater choices and control over their environment, although less specific about how such choice and control could be demonstrated through an observable metric. Alternatively, Melfi $(2013 ; 2014)$ proposed several directly testable hypotheses for an enrichment function of animal training procedures, which included training being enriching because it: (i) affords learning opportunities, and learning is considered to be enriching; (ii) can achieve the same results as enrichment; (iii) increases human-animal interactions; (iv) provides a dynamic change in the animals' day; and (v) facilitates the provision of enrichment. These hypotheses placed training itself as an independent variable, with the resulting response changes as dependent variables demonstrating an enriched outcome. Thus, although Melfi concluded that little published evidence existed at that time to demonstrate training could function as enrichment, the framework itself provided a means to achieve such results.

The following paper examines training as enrichment by first detailing a brief history of (a) modern animal training practices, with a focus on respondent and operant conditioning procedures, and (b) the use of environmental enrichment to improve behavioral welfare. Most of the paper focuses specifically on learning theory applied to zoo animals, since the history and current evidence for training as enrichment primarily occurs within this field and setting. This review proposes three means (with supporting research) by which training could function as enrichment: (1) training facilitates 
enrichment usage, (2) training modifies interactions, conspecific or otherwise, and (3) training expands behavioral repertoires. For all the above, the review examines the relevant supporting literature based on a demonstrated ability to improve behavioral welfare. In addition, the review proposes areas of research that could provide further evidence for the ability of training to function as enrichment.

\subsection{A Brief History of Modern Animal Training}

Modern animal training procedures are linked to two major events: (1) Skinner's discovery of shaping, or the differential reinforcement of successive approximations to a target response (Peterson, 2004; Pierce \& Cheney, 2013; Skinner, 1951), and (2) the creation of a field of Applied Animal Psychology by Keller and Marian Breland and based on Skinner's operant conditioning principles (Bailey \& Gillaspy, 2005; Bihm et al., 2010; Breland \& Breland, 1951). It was the latter development that propelled the field of animal training into a modern era, with the Brelands training a wide variety of animal species for commercials, coin-operated fair/zoo acts, and public shows (Curtis, 1957; Gillaspy et al., 2014; Yin, 2012). Many of these animal training procedures relied on the use of conditioned reinforcement to shape desired behavior rapidly and precisely (Dorey \& Cox, 2018; Fernandez, 2001; Ramirez, 1999).

Because of these advances in applied animal training procedures, particularly the use of positive reinforcement with marine mammals trained for public shows, zoos and other animal facilities began to explore using similar methods to train animals for routine husbandry practices. For instance, San Diego Zoo implemented a shaping protocol that allowed a diabetic drill (Mandrillus leucophaeus) to voluntarily receive insulin injections (Priest, 1991). Denver Zoo trained nyala (Tragelaphus angasi) and bongo (Tragelaphus eurycerus) to voluntarily enter crates for blood draws and other veterinary procedures (Grandin et al., 1995; Phillips et al., 1998). Bloomsmith et al. (1998) successfully used reward-based methods to train large groups of chimpanzees (Pan troglodytes) to voluntarily move (i.e., "shift") from outdoor areas to an indoor portion of their enclosures. The use of positive reinforcement-based training procedures are now commonplace in many zoos, with some organizations requiring standardized training protocols for a facility to receive accreditation (AZA, 2020; Dadone et al., 2016; EAZA, 2019; Mackie, 2020; Savastano et al., 2003; Young \& Cipreste, 2004).

Thus, modern animal training procedures have had the benefit of giving animals the choice to actively participate in interactions that result in improved veterinary care. However, using training to improve husbandry practices, and therefore the physiological welfare of an animal, is fundamentally different than saying that training itself is enriching. This distinction is why testable hypotheses and data-driven results are necessary for demonstrating whether training functions as a form of enrichment.

\subsection{A Brief History of Environmental Enrichment}

The use of environmental enrichment in zoos can be traced to Hal Markowitz (1978; 1982), who served as Director of the Oregon Zoological Research Center and Associate Director of the Portland/Washington Park Zoo (now the Oregon Zoo). While prior work 
in zoos and similar settings described the need for promoting the well-being of captive animals (Breland, 1962; Hediger, 1950; Yerkes, 1925), Markowitz and his colleagues were the first to promote a systematic, functional approach to the behavior of zoo animals through behavioral engineering (Markowitz et al., 1978; Markowitz \& Stevens, 1978; Schmidt \& Markowitz, 1977). The term "behavioral engineering" itself was taken directly from the application of Skinner's operant conditioning procedures, or the field of Applied Behavior Analysis (Capshew,1993; Homme et al., 1968). Through the creation of contrived, reinforcement-based learning contingencies, Markowitz and his colleagues were able to produce mechanical levers that would require white-handed gibbons (Hylobates lar) to swing across their enclosure to activate the levers and receive a food reward, mandrills (Mandrillus sphinx) to compete against zoo visitors in a computerized arcade-like reaction game, and polar bears (Ursus maritimus) to vocalize into a voiceoperated relay system that would result in a fish being catapulted into their exhibit (Markowitz, 1978; 1982). All the above was done to produce desired behaviors (e.g., swinging) or reduce undesired responses (e.g., begging) as a form of artificial, mechanized "occupational therapy" for the zoo animals.

Among the criticisms of such applications were the artificiality of the procedures involved, as well as the inherent problems of mechanical apparatus being costly or requiring constant maintenance (Hancocks, 1980; Hutchins et al., 1984). Nonetheless, these enrichment practices produced an empirical approach to all aspects of exhibiting animals, including animal behavior, exhibit design, and visitor perception and behavior (Bitgood \& Patterson, 1987; Coe, 1985; Forthman-Quick, 1984; Finlay et al., 1987; Maple \& Finlay, 1986; Maple \& Finlay, 1987; Markowitz \& Spinelli, 1986). Environmental enrichment as a modern practice would emerge, where all features of how an animal interacted with their environment would be examined for its welfare benefits (Hoy et al., 2010; Mellen \& MacPhee, 2001; Mench, 1998; Shepherdson, 1998). This in turn would inspire several books dedicated to the concept of environmental enrichment for animals under human care, including zoos, labs, farms, and with pets (Bender \& Strong, 2019; Markowitz, 2011; Shepherdson et al., 1998; Young, 2003).

The result is that environmental enrichment is now synonymous with changes that produce an observable, measurable improved state of well-being for an animal (Newberry, 1995; Mellen \& MacPhee, 2001; Shepherdson et al., 1998). Enrichment should therefore be defined as the interaction (i.e., contingency) between a response and a stimulus or event, not simply the object or event. In the case of training as enrichment, this must be demonstrated whereby the training procedure produces an observable, measurable enriched outcome. In other words, for training to be shown to be enriching, we must demonstrate that training itself enriched the welfare of the animal.

\subsection{Training as Enrichment - Literature Review and Categories}

To conduct the literature review, two databases were searched: Google Scholar ${ }^{\mathrm{TM}}$ and Web of Science. Search terms were "husbandry training" OR "animal training" AND "environmental enrichment" OR "behavioral enrichment" AND "animal welfare". Searches were limited to articles published in English and were not limited by year of 
publication. The literature search yielded 636 results. Following a review of titles and abstracts, the search was narrowed to 114 results. Papers were restricted to studies that directly measured behavior and incorporated a control (non-training) condition compared to at least one training condition. This produced 33 papers that attempted to empirically examine the effects of training on captive behavioral welfare.

The following section details how training could function as enrichment, dividing the outcome of the literature review into three specific categories: (1) training facilitates enrichment usage, (2) training modifies interactions, conspecific or otherwise, and (3) training expands behavioral repertoires. As noted previously, for each category the paper reviews the relevant supporting literature based on demonstrated ability to improve behavioral welfare.

\section{Training Facilitates Enrichment Usage}

One of the most direct ways to demonstrate that training can function as enrichment is by using training procedures to increase interactions with enrichment devices. However, only a few studies have empirically examined this effect. In the first published research paper on the effects of enrichment, Yanofsky and Markowitz (1978) were able to show that two mandrills trained to compete in a reaction time activity against visitors (the game previously described, which the mandrills voluntarily participated in) resulted in a decrease in stereotypic behaviors and increase in overall exhibit use. Markowitz and LaForse (1987) were able to increase overall activity, including increased foraging/hunting behaviors, and reduce time spent inactive, by training and rewarding two African servals (Leptailurus serval) for chasing artificial prey through a constructed tube in their exhibit. Markowitz et al. (1995) were able to train and reward an African leopard (Panthera pardus pardus) for stalking and chasing naturalistic acoustic prey (e.g., bird songs) along an artificial tree trunk, which resulted in increased time spent active (primarily foraging/hunting) and visibly on exhibit, as well as decreased time spent pacing while the enrichment device was operating. Finally, Fernandez et al. (2019) were able to use an autoshaping procedure (stimulus-stimulus pairings to elicit voluntary behavior; Brown \& Jenkins, 1968) to combine training plus enrichment (i.e., training enrichment item contacts) to increase time spent swimming and interacting with the devices post-training.

As noted, training animals to interact with enrichment devices is one of the most direct ways to demonstrate that training can enrich. Also, the use of computers or other technology are now readily available and can facilitate enrichment usage in the absence of direct human contact (Carter et al., 2021; Coe \& Hoy, 2020). Nonetheless, the idea that training animals to interact with enrichment appears less natural or only produces temporary increases in such enrichment interactions may contribute to why this is a less common practice (Fernandez et al., 2019). Below the review discusses two indirect methods of using training procedures to facilitate enrichment interactions. 


\subsection{Contrafreeloading and Enrichment}

Contrafreeloading describes the phenomenon where animals will choose to work for food (e.g., press a lever or operate similar operandum) over freely available food (Inglis et al., 1997; Jensen, 1963). The phenomenon has been observed across several different species and settings, including labs, farms, and zoos (De Jonge et al., 2008; Jensen et al., 2002; Lindqvist \& Jensen, 2009; Neuringer, 1969; Ogura, 2011; Young \& Lawrence, 2003). However, only a few studies have examined the contrafreeloading effect with respect to environmental enrichment. McGowan et al. (2010) were able to demonstrate that captive grizzly bears (Ursus arctos horribilis) would spend at least a portion of their time retrieving food from ice blocks or enrichment boxes over free food alone. Vasconcellos et al. (2012) showed that captive maned wolves (Chrysocyon brachyurus) would spend more time searching for food scattered across vegetation, as well as consume approximately half their diet from scattered feedings when compared to food delivered on a tray in one section of their enclosures. Sasson-Yenor and Powell (2019) demonstrated that several zoo-housed giraffes (Giraffa camelopardalis) were more likely to contrafreeload when presented simultaneously with easily accessed or more time-consuming enrichment foraging devices. These studies provide indirect support that working for food, a similar activity to many training procedures, can provide more enriching opportunities for animals.

\subsection{Preference Assessments and Enrichment}

The use of paired-choice preference assessments, where choices of potential rewards are systematically evaluated by presenting all possible pairs to an animal for their selection, were first used in zoos almost two decades ago (Fernandez et al., 2004). Since then, several studies have used similar preference assessments to evaluate their ability to determine potential enrichment activities/items (Clayton \& Shrock, 2020; Fernandez \& Timberlake, 2005; Fernandez \& Timberlake, 2019b; Mehrkam \& Dorey, 2014; Woods et al., 2020). In one study, Dorey et al. (2015) were able to demonstrate that two of their four subjects, zoo-housed wolves (Canis lupus and Canis lupus arctos), preferred training activities over enrichment items. Thus, all the above preference studies provide indirect evidence that a trained preference assessment could result in animals selecting more optimal enrichment activities. Furthermore, the latter Dorey et al. study directly demonstrated that training procedures could, at least for some animals, function as the most preferred form of environmental enrichment.

\section{Training Modifies Interactions, Conspecifics or Otherwise}

Training procedures are presumed to be an important component for improving the interactions animals have with conspecifics and their human trainers. For instance, with companion animals, training procedures, including type of training method used, play an important role in decreasing aggression, minimizing problem behaviors, or otherwise promoting proper dog-dog and dog-human interactions (for examples, see Batt et al., 2008; Blackwell et al., 2008; China et al., 2020; Haug, 2008; Rooney \& Cowan, 2011). Regardless, only a few studies in any animal setting have experimentally 
examined the effect of training procedures to promote social interactions that lead to enriched welfare outcomes. Bloomsmith et al. (1994) were able to effectively use a positive reinforcement training procedure to reduce aggression during feeding times in group-housed chimpanzees (Pan troglodytes). Schapiro et al. (2001) showed positive reinforcement training increased the affiliative behaviors outside of training sessions for otherwise less social rhesus macaques (Macaca mulatta). Pomerantz and Terkel (2009) used positive reinforcement to increase chimpanzee prosocial behaviors (e.g., grooming and playing) outside of training sessions. Carrasco et al. (2009) rewarded play behaviors in a group of zoo-housed lowland gorillas (Gorilla gorilla gorilla) and demonstrated an increase in affiliative behaviors and a decrease in aggression as a result. Spiezio et al. $(2016$; 2017) trained individuals to enter a separate testing area and were able to show an increase in affiliative behaviors and decrease in aggression with zoo-housed vervet monkeys (Chlorocebus aethiops) and ring-tailed lemurs (Lemur catta), respectively. These studies provide support for the concept that training can function as enrichment by modifying the interactions animals have with their conspecifics. A sub-section of the use of training as enrichment through human-animal interactions is considered below.

\subsection{Human-Animal Interactions and Enrichment}

Human-Animal Interactions (HAI) have become an increasingly popular topic of study for applied animal behavior research. A subset of this area of research has focused on both positive and negative HAIs as Animal-Visitor Interactions (AVIs) observed in zoos (for reviews, see Davey, 2007; Fernandez et al., 2009; Godinez \& Fernandez, 2019; Hosey, 2000; Sherwen \& Hemsworth, 2019). More recently, the topic of HAIs as a form of enrichment, with an emphasis on zoos, has been discussed (Claxton, 2011; Melfi, 2013). Here the review focuses specifically on the use of training procedures that result in both increased HAIs, as well as a demonstrated enriched effect on the behavioral welfare of those animals.

Anderson et al. (2003) found that the use of an animal training demonstration with zoo-housed Asian small-clawed otters (Aonyx cinereus) increased the overall otter activity levels (a measure directly associated with enriched animal welfare), as well as increased overall visitor perceptions and stay times at the exhibit. Ward and Melfi (2013) demonstrated that multiple species of zoo-housed animals trained using positive reinforcement had lower latencies to keeper cues (e.g., being asked to move on- or offexhibit), a measure they associated with less fear of humans. Leeds et al. (2016) showed positive reinforcement training with a zoo-housed lowland gorilla led to a reduction in aggression to keepers in the times following training sessions. Fernandez et al. (2021b) found that training zoo-housed Asian and African elephants (Elephas maximus and Loxodonta africana) to engage in public feedings were correlated with increased social interactions with the keepers/visitors, increased foraging, decreased inactivity, and decreased stereotypic behaviors when compared to non-public feed days, as well as the times following a public feeding. Therefore, all the above results provide evidence that training procedures can modify HAIs to enrich the welfare of the trained animals. 


\section{Training Expands Behavioral Repertoires}

The idea that training produces new behaviors that result in enriched welfare for those animals is the broadest category of the three described within this paper, and likewise, one of the more difficult to empirically demonstrate. Melfi (2013) described three separate hypotheses, all of which could contribute to training functioning as enrichment through such a phenomenon: (1) training affords learning, and learning is considered enriching, (2) training achieves the same results as enrichment, and (3) training provides a dynamic change in the animals' day. Since they are all related, the review focuses on the more broadly described category that encompasses all three of these hypotheses: Training functions as enrichment by expanding behavioral repertoires.

In Melfi's (2013) section on training being enriching because it achieves the same results as traditional environmental enrichment, she suggests two papers that further this concept. Hare and Sevenich (2001) provide an example of increasing tiger (Panthera tigris) tree scratching through enrichment or training. In the enrichment example, multiple scents are placed on a deadfall tree in the tigers' exhibit. In the training example, the tigers are rewarded for scratching the tree. The authors propose the same result of tree scratching could be obtained through either enrichment or training, although no data are presented to make such a direct comparison. Melfi also describes a study in which training and enrichment conditions for two zoo-housed elephants are directly compared (McCormick \& Melfi, 2003; as cited in Melfi, 2013). The aim of the study was to increase behavioral diversity in the elephants, with the result being that enrichment increased behavioral diversity for one of the elephants, but training had no effect. Thus, while both papers provide a conceptual framework and method for making such training and environmental enrichment comparisons, neither provide evidence that training can produce similar effects to enrichment.

In a study by Pryor et al. (1969), a rough-toothed dolphin (Steno bredanensis) was rewarded for engaging in a novel response not previously trained. During experimental sessions, a context cue (e.g., rung bell) was provided to indicate that only new, previously non-rewarded responses would be rewarded. During the study, the dolphin engaged in at least four distinct, novel responses. Pryor and Chase (2014) expand on the importance of these findings, including the use of training/shaping for creating behavioral variability. Behavioral variability is an important welfare/enrichment measure, as expanded repertoires can be measured more directly through such variability via behavioral diversity (see Discussion). Therefore, while the Pryor et al. study does not provide evidence that training animals to engage in novel behavior enriches their welfare, it is an important point to consider when examining evidence for training functioning as enrichment through expanded behavioral repertoires.

Two studies have examined the effects of training on the adoptability and behaviors presented in shelter animals. Luescher and Medlock (2009) examined the effects of training on the adoption rates of domestic shelter dogs (Canis lupus). Through a combination of halter and clicker training (e.g., walking on a leash, approaching people without jumping on them, or sitting on command), the 92 dogs trained were 1.4 times 
more likely to be adopted than the non-trained control dogs. In a similar study, Grant and Warrior (2019) examined the effects of clicker training domestic shelter cats (Felis catus). As a result of clicker training, all 12 cats increased their time spent exploring, at the front of their enclosure, and being able to be contacted, as well as decreased their time spent inactive. Combined, these two studies suggest that training can expand behavioral repertoires not only to improve behavioral welfare (and thus, enrich), but also to increase the likelihood of a shelter animal being adopted. While indirectly related to behavior, the ability for many shelter animals to avoid being euthanized is dependent on their adoptability, and as a result is one of the most important welfare-related measures.

These studies provide partial evidence that training procedures can functionally enrich by expanding behavioral repertoires. While greater testing is necessary, particularly with respect to direct comparisons of training and traditional environmental enrichment conditions, there is moderate support for the use of training to increase variability and other measures associated with behavioral welfare, and therefore have an enrichment-like effect. Below the review considers two sub-sections of this category: (1) the ability of training to reduce behaviors associated with sub-optimal welfare, and (2) the use of training in animal shows to promote the behavioral welfare of those animals.

\subsection{Training Reduces Undesired Behaviors}

Training procedures have effectively been used to minimize the occurrence of behaviors otherwise associated with deterred welfare (Bloomsmith et al., 2007). Bassett et al. (2003) found that common marmosets (Callithrix jacchus) trained to voluntarily give urine samples exhibited less stress-related responses (e.g., self-scratching and scent marking) following training and compared to non-trained marmosets. Baker et al. (2009) found that some rhesus macaques trained for husbandry procedures showed significant reductions in abnormal behaviors such as self-injury or stereotypic behaviors. Pomerantz and Terkel (2009) demonstrated positive reinforcement training with chimpanzees resulted in a reduction in abnormal and stress-related behaviors during training sessions. Coleman and Maier (2010) showed that positive reinforcement training with a group of rhesus macaques reduced stereotypic behaviors outside of training sessions. Shyne and Block (2010) found that husbandry training procedures for African wild dogs (Lycaon pictus) led to a reduction in stereotypic behaviors following training sessions. These results demonstrate that training can enrich by reducing behaviors associated with lowered behavioral welfare. The implication for decreasing such responses is that the opportunities to engage in behaviors associated with positive welfare are increased.

\subsection{Training for Animal Shows}

A final sub-section worth considering is the effect of training animals for shows as a source of potential environmental enrichment. As described earlier, the training of marine mammals for shows played an important role in the promotion of regular husbandry training procedures in zoos (see 'A Brief History of Modern Animal Training' section). The use of positive reinforcement to promote both voluntary interactions and behavioral welfare has been a hallmark for show animals (Brando, 2012; Eskelinen et al., 
2015). However, others have suggested that training animals for shows, or simply their existence as trained show animals, leads to a reduction in both the physiological and behavioral welfare of those animals (Rose \& Parsons, 2019). Only a few studies have directly investigated the results of training animals for shows or interactions on their overall welfare. Kyngdon et al. (2003) found that short-beaked common dolphin (Delphinus delphis) trained to engage in a 'Swim-with-Dolphins' program increased their surfacing and use of outside areas during programs, but otherwise showed few behavioral changes before, during, or after the interactions. Similarly, Trone et al. (2005) found few behavioral differences in bottlenose dolphins (Tursiops truncatus) in the times before or after interaction programs, with an increase in play behaviors following interactions. Delfour et al. (2020) used Qualitative Behavioral Assessments (QBAs) to assess trainerdolphin interactions and the welfare of bottlenose dolphins located at five different European facilities. All six parameters tested in the QBA showed high levels of interest by the dolphins to interact with trainers and voluntarily participate in training sessions. Thus, preliminary evidence suggests that training some animals for and participating in shows may function as a source of enrichment for those animals.

\section{Discussion}

Training has been discussed as a form of enrichment, including the learning that may occur from interacting with potential enrichment items (Young et al., 2020). While the broader enriching and welfare value of training procedures is often assumed, this review attempts to frame the concept of training as enrichment as an empirical question, with results from previous studies considered as forms of support for this claim. If training is said to be enriching, then we must first identify how training can function as enrichment and confirm the likelihood of such possibilities through observable, measurable results. This paper considers three such categories and their supporting evidence: (1) training facilitates enrichment usage, (2) training modifies interactions, conspecific or otherwise, and (3) training expands behavioral repertoires.

Training has been demonstrated to enrich by (1) facilitating the use of enrichment, with a limited number of studies directly training animals to use enrichment devices (Fernandez et al., 2019; Markowitz \& LaForse, 1987; Markowitz et al., 1995; Yanofsky \& Markowitz, 1978). In addition, animals demonstrating a desire to 'work for food' and animals selecting items in preference assessments to be delivered as enrichment both provide further evidence for training facilitating enrichment usage. For instance, Dorey et al.'s (2015) implementation of preference assessments to allow wolves to select between traditional environmental enrichment or training procedures is a successful demonstration of how both scientists and practitioners can evaluate the potential enriching function of any training procedure.

Training has also been demonstrated to enrich by (2) modifying interactions, conspecific or otherwise, with several studies demonstrating that training can result in decreased aggression and increased affiliation in primates (Bloomsmith et al., 1994; Carrasco et al., 2009; Pomerantz \& Terkel, 2009; Schapiro et al., 2001; Spiezio et al., 2016; Spiezio et al., 2017). Additionally, training has been used to modify human-animal 
interactions (HAIs), which is important because (a) HAIs are an emerging field of interest for applied animal behavior research, and (b) past research has demonstrated that HAIs can be a source of stress or otherwise result in decreased behavioral welfare for many animals. In one study, Fernandez et al. (2021b) were able to train elephants to interact with keepers and visitors during public feeds, which resulted in increased keeper/visitor interactions, increased foraging, decreased inactivity, and decreased stereotypic behaviors. Thus, using training through public feedings or similar interactions is an effective way to demonstrate the enriching value of animal training procedures.

Finally, training has been suggested to enrich by (3) expanding behavioral repertoires, with several studies showing that training can increase behavioral variability, increase behaviors associated with positive welfare, and/or decrease undesired behaviors (Baker et al., 2009; Bassett et al., 2003; Bloomsmith et al., 2007; Coleman \& Maier, 2010; Grant \& Warrior, 2019; Hare \& Sevenich, 2001; Leuscher \& Medlock, 2009; McCormick \& Melfi, 2003; Pomerantz \& Terkel, 2009; Pryor et al., 1969; Shyne \& Block, 2010). Training as enrichment also appears to play an important role in animal shows, with Delfour et al. (2020) effectively demonstrating that dolphins displayed high interest in both participating in shows and interacting with trainers. The use of training within animal shows is one of the most important illustrations of the training as enrichment effect, since it involves an animal's willingness to 'work for food', desire to interact with trainers, and ultimately, whether the show itself produces an overall improvement in behavioral welfare.

Taken together, the studies reviewed in this paper support the assertion that training can function as enrichment. It is hoped that this review also provides a source of inspiration for more research on the use of training as a form of enrichment, with multiple areas requiring more detailed investigation. For instance, there is needed research to evaluate training animals to engage with potential enrichment devices in meaningful ways, to assess public feedings or similar HAIs as enrichment procedures, and to determine the effects of animal shows on the welfare of those animals. Likewise, there is greater interest in assessing the welfare of animals through positive rather than negative indices, as one of the main goals of animal welfare research is to optimize overall welfare, rather than simply provide adequate outcomes (Lawrence et al., 2019; Mellor, 2016; Mellor \& Beausoleil, 2019; Mellor et al., 2020). As such, three additional areas of emphasis are considered below, with a focus on how they could expand our current knowledge of the training as enrichment effect.

\subsection{Behavioral Diversity and Enclosure Use Variability}

As noted above, there is greater interest in positive welfare metrics to assess the well-being of animals. The use of behavioral diversity and enclosure use variability indices are examples of such methods (see Brereton, 2020; Miller et al., 2020 for reviews). Identifying variability in both the frequency of behaviors and areas of an enclosure used by an animal are directly relevant to whether training functions as enrichment, since training could be hypothesized to have a positive or negative effect on both measures. For instance, does training only a select number of behaviors result in an 
increase or decrease in the variability of those behaviors? Does training in limited areas increase or decrease overall enclosure use? It is possible that, for training to function as enrichment, we need to take into consideration both the frequency and type of behaviors we train, as well as the regularity or variability in the places where we train.

\subsection{The Learning Effect}

Training procedures offer a source of learning/enrichment opportunities not just for the trainee(s), but for the trainer(s) and those observing training sessions as well (Fernandez \& Timberlake, 2008; Hazel et al., 2015; Lukas et al., 1998). Students, volunteers, and visitors have the potential to function as a source of training enrichment themselves, provided they are somehow involved with training interactions. Similarly, the type and regularity of such training interactions could be the source of independent variables within any training as enrichment study. With the increased interest in HAI research and the desire for many visitors at various animal facilities to interact with the animals, there is a near unlimited source of possibilities to consider for future research.

\subsection{Within-Subject Methodology}

The field of Behavior Analysis has been integral in developing the use of husbandry training procedures and environmental enrichment for animal welfare research and practice (see both 'Brief History' sections). Behavior Analysis has also focused on within-subject methodology to implement both basic and applied research, which has resulted in many training and enrichment studies using similar designs (Alligood et al., 2017; Fernandez \& Timberlake, 2008; Maple \& Segura, 2015). Some of the many benefits of within-subject methodology include (a) a focus on many data points from a few individuals (as opposed to few data points from many individuals), (b) an emphasis on inductive data collection that modifies procedures based on real-time results (as opposed to a priori hypothesis testing), and (c) the ability to assess an individual's learning repeatedly and over time (as opposed to pre- versus post-test analyses) (Bailey \& Burch, 2017; Johnston \& Pennypacker, 2010). To properly study the training as enrichment effect, within-subject methodological designs appear best suited to address many of the possible research questions. Put simply, training is a learning-related phenomenon, and learning is often best understood by frequently measuring the performance of individuals.

\section{Conclusions}

The concept of training as enrichment has played an important role in promoting the use of training procedures as a common behavioral welfare practice. While this concept is critical for animal training practitioners, it has remained a source of speculation for those interested in its scientific validity. This review attempts to address this concept empirically by treating training as an independent variable manipulation and the enriched outcome as a dependent variable result. Evidence from existing published research supports the ability of training to facilitate enrichment usage, modify interactions, and expand behavioral repertoires in ways that enrich the welfare of those 
animals. Future research is necessary to expand our understanding of the conditions under which training might function as enrichment, as well as provide more extensive support for the notion that training procedures can be a desired, enriching activity for the lives of animals under human care. 


\section{Author Note}

Eduardo J. Fernandez (DiD https://orcid.org/0000-0001-5444-6604

I have no conflicts of interest to disclose.

This review was written while the author waited on a move to Australia from the U.S. during a global pandemic. The author would like to thank Allie Bender, Jon Coe, Stephanie Edlund, and Susan Hazel for some useful suggestions, as well as Bob Bailey and Gary Priest for help obtaining some of the articles cited within this paper.

Correspondence concerning this publication should be addressed to Eduardo J. Fernandez, School of Animal and Veterinary Sciences, The University of Adelaide, Adelaide SA 5005 AUSTRALIA. Email: edjfern@gmail.com 


\section{References}

Alligood, C. A., Dorey, N. R., Mehrkam, L. R., \& Leighty, K. A. (2017). Applying behavior-analytic methodology to the science and practice of environmental enrichment in zoos and aquariums. Zoo Biology, 36(3), 175-185.

Anderson, U. S., Kelling, A. S., Pressley-Keough, R., Bloomsmith, M. A., \& Maple, T. L. (2003). Enhancing the zoo visitor's experience by public animal training and oral interpretation at an otter exhibit. Environment and Behavior, 35(6), 826-841.

Andrews, N. L., \& Ha, J. C. (2014). The effects of automated scatter feeders on captive grizzly bear activity budgets. Journal of Applied Animal Welfare Science, 17, 148-156.

Association of Zoos and Aquariums (2020, November 20). Animal Care Manuals. https://www.aza.org/animal-care-manuals

Bailey, J. S., \& Burch, M. R. (2017). Research Methods in Applied Behavior Analysis. Routledge.

Bailey, R. E., \& Gillaspy, J. A. (2005). Operant psychology goes to the fair: Marian and Keller Breland in the popular press, 1947-1966. The Behavior Analyst, 28(2), 143-159.

Baker, K. C., Bloomsmith, M., Neu, K., Griffis, C., Maloney, M., Oettinger, B., Schoof, V. A. M., \& Martinez, M. (2009). Positive reinforcement training moderates only high levels of abnormal behavior in singly housed rhesus macaques. Journal of Applied Animal Welfare Science, 12(3), 236-252.

Bashaw, M. J., Gibson, M. D., Schowe, D. M., \& Kucher, A. S. (2016). Does enrichment improve reptile welfare? Leopard geckos (Eublepharis macularius) respond to five types of environmental enrichment. Applied Animal Behaviour Science, 184, 150-160.

Bassett, L., Buchanan-Smith, H. M., McKinley, J., \& Smith, T. E. (2003). Effects of training on stress-related behavior of the common marmoset (Callithrix jacchus) in relation to coping with routine husbandry procedures. Journal of Applied Animal Welfare Science, 6(3), 221-233.

Batt, L., Batt, M., Baguley, J., \& McGreevy, P. (2008). The effects of structured sessions for juvenile training and socialization on guide dog success and puppy-raiser participation. Journal of Veterinary Behavior, 3(5), 199-206.

Bender, A., \& Strong, E. (2019). Canine Enrichment for the Real World. Dogwise Publishing. 
Bihm, E. M., Gillaspy, J. A., Lammers, W. J., \& Huffman, S. P. (2010). IQ Zoo and teaching operant concepts. The Psychological Record, 60(3), 523-526.

Bitgood, S., \& Patterson, D. (1987). Principles of exhibit design. Visitor Behavior, 2(1), 4-6.

Blackwell, E. J., Twells, C., Seawright, A., \& Casey, R. A. (2008). The relationship between training methods and the occurrence of behavior problems, as reported by owners, in a population of domestic dogs. Journal of Veterinary

Behavior, 3(5), 207-217.

Bloomsmith, M. A., Laule, G. E., Alford, P. L., \& Thurston, R. H. (1994). Using training to moderate chimpanzee aggression during feeding. Zoo Biology, 13(6), 557-566.

Bloomsmith, M. A., Marr, M. J., \& Maple, T. L. (2007). Addressing nonhuman primate behavioral problems through the application of operant conditioning: Is the human treatment approach a useful model? Applied Animal Behaviour Science, 102(3-4), 205-222.

Bloomsmith, M. A., Stone, A. M., \& Laule, G. E. (1998). Positive reinforcement training to enhance the voluntary movement of group-housed chimpanzees within their enclosures. Zoo Biology, 17(4), 333-341.

Brando, S. I. (2012). Animal learning and training: implications for animal welfare. Veterinary Clinics: Exotic Animal Practice, 15(3), 387-398.

Breland, K. (1962). New strides in animal psychology bring about naturalistic behavior exhibits. Parks and Recreation, 45, 80.

Breland, K., \& Breland, M. (1951). A field of applied animal psychology. American Psychologist, 6(6), 202.

Brereton, J. E. (2020). Directions in animal enclosure use studies. Journal of Zoo and Aquarium Research, 8(1), 1-9.

Brown, P. L., \& Jenkins, H. M. (1968). Auto-shaping of the pigeon's key-peck. Journal of the Experimental Analysis of Behavior, 11(1), 1-8.

Capshew, J. H. (1993). Engineering behavior: project pigeon, World War II, and the conditioning of BF Skinner. Technology and Culture, 34(4), 835-857.

Carlstead, K., Brown, J. L., \& Seidensticker, J. (1993). Behavioral and adrenocortical responses to environmental changes in leopard cats (Felis bengalensis). Zoo Biology, 12, 321-331. 
Carlstead, K., \& Seidensticker, J. (1991). Seasonal variation in stereotypic pacing in an American black bear (Ursus americanus). Behavioural Processes, 25, 155-161.

Carlstead, K., Seidensticker, J., \& Baldwin, R. (1991). Environmental enrichment for zoo bears. Zoo Biology 10, 3-16.

Carrasco, L., Colell, M., Calvo, M., Abello, M. T., Velasco, M., \& Posada, S. (2009). Benefits of training/playing therapy in a group of captive lowland gorillas (Gorilla gorilla gorilla). Animal Welfare, 18(1), 9-19.

Carter, M., Sherwen, S., \& Webber, S. (2021). An evaluation of interactive projections as digital enrichment for orangutans. Zoo Biology, 40(2), 107-114

Coe, J., \& Hoy, J. (2020). Choice, control and computers: Empowering wildlife in human care. Multimodal Technologies and Interaction, 4(4), 92

China, L., Mills, D. S., \& Cooper, J. J. (2020). Efficacy of Dog Training With and Without Remote Electronic Collars vs. a Focus on Positive Reinforcement. Frontiers in Veterinary Science, 7, 508.

Claxton, A. M. (2011). The potential of the human-animal relationship as an environmental enrichment for the welfare of zoo-housed animals. Applied Animal Behaviour Science, 133(1-2), 1-10.

Clayton, M., \& Shrock, T. (2020). Making a Tiger's Day: Free-Operant Assessment and Environmental Enrichment to Improve the Daily Lives of Captive Bengal Tigers (Panthera tigris tigris). Behavior Analysis in Practice, 1-11.

Coe, J. C. (1985). Design and perception: Making the zoo experience real. Zoo Biology, 4(2), 197-208.

Coe, J. (2004). 'Mixed species rotation exhibits,' Jon Coe Design Pty Ltd. (originally prepared for ARAZPA 2004 Annual Conference).

Coleman, K., \& Maier, A. (2010). The use of positive reinforcement training to reduce stereotypic behavior in rhesus macaques. Applied Animal Behaviour Science, 124(3-4), 142-148.

Curtis, L. (1957). Coin-operated animal acts. Parks and Recreation, 40, 18.

Dadone, L. I., Schilz, A., Friedman, S. G., Bredahl, J., Foxworth, S., \& Chastain, B. (2016). Training giraffe (Giraffa camelopardalis reticulata) for front foot radiographs and hoof care. Zoo Biology, 35(3), 228-236. 
Davey, G. (2007). Visitors' effects on the welfare of animals in the zoo: A review. Journal of Applied Animal Welfare Science, 10(2), 169-183.

De Jonge, F. H., Tilly, S. L., Baars, A. M., \& Spruijt, B. M. (2008). On the rewarding nature of appetitive feeding behaviour in pigs (Sus scrofa): do domesticated pigs contrafreeload? Applied Animal Behaviour Science, 114(3-4), 359-372.

Desmond, T., \& Laule, G. (1994). Use of positive reinforcement training in the management of species for reproduction. Zoo Biology, 13(5), 471-477.

Domjan, M. P. (2014). The Principles of Learning and Behavior. Cengage Learning.

Dorey, N. R., \& Cox, D. J. (2018). Function matters: a review of terminological differences in applied and basic clicker training research. PeerJ, 6, e5621.

Dorey, N. R., Mehrkam, L. R., \& Tacey, J. (2015). A method to assess relative preference for training and environmental enrichment in captive wolves (Canis lupus and Canis lupus arctos). Zoo Biology, 34(6), 513-517.

Eskelinen, H. C., Winship, K. A., \& Borger-Turner, J. L. (2015). Sex, age, and individual differences in bottlenose dolphins (Tursiops truncatus) in response to environmental enrichment. Animal Behavior and Cognition, 2(3), 241-253.

European Association of Zoos and Aquaria (2019, April 25). Standards for the accommodation and care of animals in zoos and aquaria. https://www.eaza.net/assets/Uploads/Standards-and-policies/2019-04-EAZAStandards-for-Accomodation-and-Care.pdf

Fernandez, E. J. (2001). Click or treat: A trick or two in the zoo. American Animal Trainer Magazine, 2(2), 41-44.

Fernandez, E. J. (2010). Stereotypies and Foraging: Appetitive Search Behaviors and Stereotypies in Captive Animals. VDM Publishing.

Fernandez, E. J. (2021). Appetitive search behaviors and stereotypies in polar bears (Ursus maritimus). Behavioural Processes, 182, 104299. https://doi.org/10.1016/j.beproc.2020.104299

Fernandez, E. J., Dorey, N., \& Rosales-Ruiz, J. (2004). A two-choice preference assessment with five cotton-top tamarins (Saguinus oedipus). Journal of Applied Animal Welfare Science, 7(3), 163-169.

Fernandez, E. J., Kinley, R. C., \& Timberlake, W. (2019). Training penguins to interact with enrichment devices for lasting effects. Zoo Biology, 38(6), 481-489. 
Fernandez, E. J., Myers, M., \& Hawkes, N. C. (2021a). The effects of live feeding on swimming activity and exhibit use in zoo Humboldt penguins (Spheniscus humboldti). Journal of Zoological and Botanical Gardens, 2(1), 88-100. https://doi.org/10.3390/jzbg2010007

Fernandez, E. J., Tamborski, M. A., Pickens, S. R., \& Timberlake, W. (2009). Animalvisitor interactions in the modern zoo: Conflicts and interventions. Applied Animal Behaviour Science, 120(1-2), 1-8.

Fernandez, E.J., \& Timberlake, W. (2005, May). The Functional Value of Enrichment: Determining Environmental Enrichment Effects in Lemurs through the Use of Paired-Choice Preference Assessments. Paper presented at the Association for Behavior Analysis Conference, Chicago, IL (USA).

Fernandez, E. J., \& Timberlake, W. (2008). Mutual benefits of research collaborations between zoos and academic institutions. Zoo Biology, 27(6), 470-487.

Fernandez, E. J., \& Timberlake, W. (2019a). Foraging devices as enrichment in captive walruses (Odobenus rosmarus). Behavioural Processes, 168, 103943.

Fernandez, E. J., \& Timberlake, W. (2019b). Selecting and testing environmental enrichment in lemurs. Frontiers in Psychology, 10, 2119.

Fernandez, E. J., Upchurch, B., \& Hawkes, N. C. (2021b). Public feeding interactions as enrichment for three zoo-housed elephants. Animals, 11(6), 1689. https://doi.org/10.3390/ani11061689

Finlay, T., James, L. R., \& Maple, T. L. (1988). People's perceptions of animals: the influence of zoo environment. Environment and Behavior, 20(4), 508-528.

Forthman-Quick, D. L. (1984). An integrative approach to environmental engineering in zoos. Zoo Biology, 3, 65-77.

Forthman, D. L., \& Ogden, J. J. (1992). The role of applied behavior analysis in zoo management: today and tomorrow. Journal of Applied Behavior Analysis, 25(3), 647.

Gillaspy Jr, J. A., Brinegar, J. L., \& Bailey, R. E. (2014). Operant psychology makes a splash - in marine mammal training (1955-1965). Journal of the History of the Behavioral Sciences, 50(3), 231-248.

Godinez, A. M., \& Fernandez, E. J. (2019). What is the zoo experience? How zoos impact a visitor's behaviors, perceptions, and conservation efforts. Frontiers in Psychology, 10, 1746. 
Graham, L., Wells, D. L., \& Hepper, P. G. (2005). The influence of olfactory stimulation on the behaviour of dogs housed in a rescue shelter. Applied Animal Behaviour Science, 91, 143-153.

Grandin, T., Rooney, M. B., Phillips, M., Cambre, R. C., Irlbeck, N. A., \& Graffam, W. (1995). Conditioning of nyala (Tragelaphus angasi) to blood sampling in a crate with positive reinforcement. Zoo Biology, 14(3), 261-273.

Grant, R. A., \& Warrior, J. R. (2019). Clicker training increases exploratory behaviour and time spent at the front of the enclosure in shelter cats; Implications for welfare and adoption rates. Applied Animal Behaviour Science, 211, 77-83.

Hancocks, D. (1980). Bringing nature into the zoo: inexpensive solutions for zoo environments. International Journal for the Study of Animal Behavior Problems, $1,170-177$.

Hare, V.J., Sevenich, M. (2001). Is it training or is it enriching? In Proceedings of the Fourth International Conference on Environmental Enrichment, Edinburgh, Scotland.

Haug, L. I. (2008). Canine aggression toward unfamiliar people and dogs. Veterinary Clinics of North America: Small Animal Practice, 38(5), 1023-1041.

Hazel, S. J., O'Dwyer, L., \& Ryan, T. (2015). "Chickens are a lot smarter than I originally thought": changes in student attitudes to chickens following a chicken training class. Animals, 5(3), 821-837.

Hediger, H. (1950). Wild Animals in Captivity. Butterworths Scientific Publications.

Homme, L., C'de Baca, P., Cottingham, L., \& Homme, A. (1968). What behavioral engineering is. The Psychological Record, 18(3), 425-434.

Hosey, G. R. (2000). Zoo animals and their human audiences: what is the visitor effect? Animal Welfare, 9(4), 343-357.

Hoy, J. M., Murray, P. J., \& Tribe, A. (2010). Thirty years later: Enrichment practices for captive mammals. Zoo Biology, 29(3), 303-316.

Hutchins, M., Hancocks, D., \& Crockett, C. (1984). Natural solutions to the behavioral problems of captive animals. Zoologische Garten, 54, 28-42.

Inglis, I. R., Forkman, B., \& Lazarus, J. (1997). Free food or earned food? A review and fuzzy model of contrafreeloading. Animal Behaviour, 53(6), 1171-1191. 
Jensen, G. D. (1963). Preference for bar pressing over" freeloading" as a function of number of rewarded presses. Journal of Experimental Psychology, 65(5), 451.

Jensen, P., Schütz, K., \& Lindqvist, C. (2002). Red jungle fowl have more contrafreeloading than white leghorn layers: effect of food deprivation and consequences for information gain. Behaviour, 139(9), 1195-1209.

Johnston, J. M., \& Pennypacker, H. S. (2010). Strategies and Tactics of Behavioral Research. Routledge.

Kyngdon, D. J., Minot, E. O., \& Stafford, K. J. (2003). Behavioural responses of captive common dolphins Delphinus delphis to a 'Swim-withDolphin'programme. Applied Animal Behaviour Science, 81(2), 163-170.

Laule, G. E. (2003). Positive reinforcement training and environmental enrichment: enhancing animal well-being. Journal of the American Veterinary Medical Association, 223(7), 969-973.

Laule, G. E., Bloomsmith, M. A., \& Schapiro, S. J. (2003). The use of positive reinforcement training techniques to enhance the care, management, and welfare of primates in the laboratory. Journal of Applied Animal welfare Science, 6(3), 163-173.

Laule, G., \& Desmond, T. (1998). Positive reinforcement training as an enrichment strategy. In Second Nature: Environmental Enrichment for Captive Animals, $302-313$.

Laule, G., \& Whittaker, M. (2007). Enhancing nonhuman primate care and welfare through the use of positive reinforcement training. Journal of Applied Animal Welfare Science, 10(1), 31-38.

Lawrence, A. B., Vigors, B., \& Sandøe, P. (2019). What is so positive about positive animal welfare? - a critical review of the literature. Animals, 9(10), 783.

Leeds, A., Elsner, R., \& Lukas, K. E. (2016). The effect of positive reinforcement training on an adult female western lowland gorilla's (Gorilla gorilla gorilla) rate of abnormal and aggressive behavior. Animal Behavior and Cognition, 3(2), 7887.

Lindqvist, C., \& Jensen, P. (2009). Domestication and stress effects on contrafreeloading and spatial learning performance in red jungle fowl (Gallus gallus) and White Leghorn layers. Behavioural Processes, 81(1), 80-84. 
Luescher, A. U., \& Medlock, R. T. (2009). The effects of training and environmental alterations on adoption success of shelter dogs. Applied Animal Behaviour Science, 117(1-2), 63-68.

Lukas, K. E., Marr, M. J., \& Maple, T. L. (1998). Teaching operant conditioning at the zoo. Teaching of Psychology, 25(2), 112-116.

Mackie, J. (2020). The Application of Positive Reinforcement Training to Enhance Welfare of Primates in Zoological Collections. Zoo Animal Learning and Training, 211-215.

Maple, T. L., \& Finlay, T. W. (1986). Evaluating the environments of captive nonhuman primates. In Primates (pp. 479-488). Springer.

Maple, T. L., \& Finlay, T. W. (1987). Post-occupancy evaluation in the zoo. Applied Animal Behaviour Science, 18(1), 5-18.

Maple, T. L., \& Perdue, B. M. (2013). Zoo Animal Welfare. Springer.

Maple, T. L., \& Segura, V. D. (2015). Advancing behavior analysis in zoos and aquariums. The Behavior Analyst, 38(1), 77-91.

Markowitz, H. (1978). Engineering environments for behavioral opportunities in the zoo. The Behavior Analyst, 1(1), 34.

Markowitz, H. (1982). Behavioral Enrichment in the Zoo. Van Nostrand Reinhold.

Markowitz, H. (2011). Enriching Animal Lives. Mauka Press.

Markowitz, H., Aday, C., \& Gavazzi, A. (1995). Effectiveness of acoustic "prey": Environmental enrichment for a captive African leopard (Panthera pardus). Zoo Biology, 14(4), 371-379.

Markowitz, H. \& LaForse, S. (1987). Artificial prey as behavioral enrichment devices for felines. Applied Animal Behaviour Science, 18, 31-43.

Markowitz, H., Schmidt, M. J., \& Moody, A. (1978). Behavioural engineering and animal health in the zoo. International Zoo Yearbook, 18(1), 190-194.

Markowitz, H., \& Spinelli, J. S. (1986). Environmental engineering for primates. In Primates, 489-498.

Markowitz, H., \& Stevens, V. J. (Eds.) (1978). Behavior of Captive Wild Animals. Nelson-Hall. 
McCormick, W., Melfi, V.A. (2003). How enriching is training? In Proceedings of the Sixth International Conference on Environmental Enrichment, Johannesburg, South Africa.

McGowan, R. T., Robbins, C. T., Alldredge, J. R., \& Newberry, R. C. (2010). Contrafreeloading in grizzly bears: implications for captive foraging enrichment. Zoo Biology, 29(4), 484-502.

Melfi, V. (2013). Is training zoo animals enriching? Applied Animal Behaviour Science, 147(3-4), 299-305.

Melfi, V. (2014). In response to the Letter to the Editor regarding the article: "Is training zoo animals enriching?" Applied Animal Behaviour Science, 152, 103-105.

Melfi, V. A., Dorey, N. R., \& Ward, S. J. (Eds.). (2020). Zoo Animal Learning and Training. Wiley Blackwell.

Melfi, V. A., \& Ward, S. J. (2020). Welfare implications of zoo animal training. In Zoo Animal Learning and Training, 271-288.

Mellen, J., \& Sevenich MacPhee, M. (2001). Philosophy of environmental enrichment: past, present, and future. Zoo Biology, 20(3), 211-226.

Mellor, D. J. (2016). Updating animal welfare thinking: Moving beyond the "Five Freedoms" towards "a Life Worth Living”. Animals, 6(3), 21.

Mellor, D. J., \& Beausoleil, N. J. (2019). Moving Beyond a Problem-based Focus on Poor Animal Welfare Toward Creating Opportunities to Have Positive Welfare Experiences. Mental Health and Well-being in Animals, 50.

Mellor, D. J.; Beausoleil, N. J., Littlewood, K. E., McLean, A. N., McGreevy, P. D., Jones, B., \& Wilkins, C. (2020). The 2020 Five Domains Model: Including Human-Animal Interactions in Assessments of Animal Welfare. Animals, 10, 1870 .

Mench, J. A. (1998). Environmental enrichment and the importance of exploratory behavior. In Second Nature: Environmental Enrichment for Captive Animals, 3046.

Mehrkam, L. R., \& Dorey, N. R. (2014). Is preference a predictor of enrichment efficacy in Galapagos tortoises (Chelonoidis nigra)? Zoo Biology, 33(4), 275-284.

Miller, L. J., Vicino, G. A., Sheftel, J., \& Lauderdale, L. K. (2020). Behavioral diversity as a potential indicator of positive animal welfare. Animals, 10(7), 1211. 
Neuringer, A. J. (1969). Animals respond for food in the presence of free food. Science, 166(3903), 399-401.

Newberry, R. C. (1995). Environmental enrichment: increasing the biological relevance of captive environments. Applied Animal Behaviour Science, 44(2-4), 229-243.

Ogura, T. (2011). Contrafreeloading and the value of control over visual stimuli in Japanese macaques (Macaca fuscata). Animal Cognition, 14(3), 427-431.

Peterson, G. B. (2004). A day of great illumination: BF Skinner's discovery of shaping. Journal of the Experimental Analysis of Behavior, 82(3), 317-328.

Phillips, M., Grandin, T., Graffam, W., Irlbeck, N. A., \& Cambre, R. C. (1998). Crate conditioning of bongo (Tragelaphus eurycerus) for veterinary and husbandry procedures at the Denver Zoological Gardens. Zoo Biology, 17(1), 25-32.

Pierce, W. D., \& Cheney, C. D. (2013). Behavior Analysis and Learning. Psychology Press.

Platt, D. M., \& Novak, M. A. (1997). Videostimulation as enrichment for captive rhesus monkeys (Macaca mulatta). Applied Animal Behaviour Science, 52, 139-155.

Pomerantz, O., \& Terkel, J. (2009). Effects of positive reinforcement training techniques on the psychological welfare of zoo-housed chimpanzees (Pan troglodytes). American Journal of Primatology, 71(8), 687-695.

Priest, G. (1991). Training a diabetic drill (Mandrillus leucophaeus) to accept insulin injections and venipuncture. Laboratory Primate Newsletter 30(1):1-4.

Pryor, K. (1999). Don't Shoot the Dog! Revised Edition. Bantam Books.

Pryor, K. W., \& Chase, S. (2014). Training for variability and innovative behavior. International Journal of Comparative Psychology, 27(2).

Pryor, K. W., Haag, R., \& O'Reilly, J. (1969). The creative porpoise: Training for novel behavior. Journal of the Experimental Analysis of Behavior, 12(4), 653-661.

Pryor, K., \& Ramirez, K. R. (2014). Modern animal training. In The Wiley Blackwell Handbook of Operant and Classical Conditioning, 455-482.

Ramirez, K. (1999). Animal Training: Successful Animal Management through Positive Reinforcement. Shedd Aquarium Press.

Ramirez, K. (2020). The Eye of the Trainer: Animal Training, Transformation, and Trust. First Stone Publishing. 
Rooney, N. J., \& Cowan, S. (2011). Training methods and owner-dog interactions: Links with dog behaviour and learning ability. Applied Animal Behaviour Science, 132(3-4), 169-177.

Rose, N. A., \& Parsons, E. C. M. (2019). The Case Against Marine Mammals in Captivity, $5^{\text {th }}$ Edition. Animal Welfare Institute and World Animal Protection.

Sasson-Yenor, J., \& Powell, D. M. (2019). Assessment of contrafreeloading preferences in giraffe (Giraffa camelopardalis). Zoo Biology, 38(5), 414-423.

Savastano, G., Hanson, A., \& McCann, C. (2003). The development of an operant conditioning training program for New World primates at the Bronx Zoo. Journal of Applied Animal Welfare Science, 6(3), 247-261.

Schapiro, S. J., Perlman, J. E., \& Boudreau, B. A. (2001). Manipulating the affiliative interactions of group-housed rhesus macaques using positive reinforcement training techniques. American Journal of Primatology, 55(3), 137-149.

Schmidt, M. J., \& Markowitz, H. (1977). Behavioral engineering as an aid in the maintenance of healthy zoo animals. Journal of the American Veterinary Medical Association, 171(9), 966-969.

Shepherdson, D. J. (1998). Tracing the path of environmental enrichment in zoos. In Second Nature: Environmental Enrichment for Captive Animals, 1-12.

Shepherdson, D. J., Carlstead, K., Mellen, J. D., \& Seidensticker, J. (1993). The influence of food presentation on the behavior of small cats in confined environments. Zoo Biology, 12(2), 203-216.

Sherpherdson D. J., Mellen, J. D., \& Hutchins, M., (Eds.) (1998). Second Nature: Environmental Enrichment for Captive Animals. Smithsonian Institutional Press.

Sherwen, S. L., \& Hemsworth, P. H. (2019). The visitor effect on zoo animals: Implications and opportunities for zoo animal welfare. Animals, 9(6), 366.

Sherwin, C. M., Lewis, P. D., \& Perry, G. C. (1999). The effects of environmental enrichment and intermittent lighting on the behaviour and welfare of male domestic turkeys. Applied Animal Behaviour Science, 62, 319-333.

Shyne, A., \& Block, M. (2010). The effects of husbandry training on stereotypic pacing in captive African wild dogs (Lycaon pictus). Journal of Applied Animal Welfare Science, 13(1), 56-65.

Skinner, B. F. (1951). How to teach animals. Scientific American, 185(6), 26-29. 
Spiezio, C., Piva, F., Regaiolli, B., \& Vaglio, S. (2016). Positive reinforcement training: a tool for care and management of captive vervet monkeys (Chlorocebus aethiops). Animal Welfare, 24(3), 283-290.

Spiezio, C., Vaglio, S., Scala, C., \& Regaiolli, B. (2017). Does positive reinforcement training affect the behaviour and welfare of zoo animals? The case of the ringtailed lemur (Lemur catta). Applied Animal Behaviour Science, 196, 91-99.

Trone, M., Kuczaj, S., \& Solangi, M. (2005). Does participation in Dolphin-Human Interaction Programs affect bottlenose dolphin behaviour?. Applied Animal Behaviour Science, 93(3-4), 363-374.

Vasconcellos, A. S, Adania, C. H., \& Ades, C. (2012). Contrafreeloading in maned wolves: Implications for their management and welfare. Applied Animal Behaviour Science, 140(1-2), 85-91.

Ward, S. J., \& Melfi, V. (2013). The implications of husbandry training on zoo animal response rates. Applied Animal Behaviour Science, 147(1-2), 179-185.

Wells, D. L., \& Irwin, R. M. (2008). Auditory stimulation as enrichment for zoo-housed Asian elephants (Elephas maximus). Animal Welfare, 17, 335-340.

Westlund, K. (2014). Training is enrichment — and beyond. Applied Animal Behaviour Science, 152, 1-6.

Woods, J. M., Lane, E. K., \& Miller, L. J. (2020). Preference assessments as a tool to evaluate environmental enrichment. Zoo Biology, 39(6), 382-390.

Yanofsky, R., \& Markowitz, H. (1978). Changes in general behavior of two mandrills (Papio sphinx) concomitant with behavioral testing in the zoo. The Psychological Record, 28(3), 369-373.

Yerkes, R. M. (1925). Almost Human. Century.

Yin, S. (2012). Bells and whistles: When operant conditioning clicked (and clucked). Barks Magazine, 69, 74-78.

Young, R. J. (2003). Environmental Enrichment for Captive Animals. Blackwell Publishing.

Young, R. J., de Azevedo, C. S., \& Cipreste, C. F. (2020). Environmental enrichment: the creation of opportunities for informal learning. Zoo Animal Learning and Training, 101-118. 
Young, R. J., \& Cipreste, C. F. (2004). Applying animal learning theory: training captive animals to comply with veterinary and husbandry procedures. Animal Welfare, 13(2), 225-232.

Young, R. J., \& Lawrence, A. B. (2003). Do domestic pigs in controlled environments contrafreeload? Journal of Applied Animal Welfare Science, 6(4), 309-318. 